\title{
La importancia de las habilidades blandas para la docencia universitaria en el contexto actual
}

doi: $10.33264 /$ rpa.201901-07

Dra. Andrea Naranjo Lama

Doctora en Psicología, Universidad Complutense de Madrid

\section{Resumen}

Este ensayo se enmarca dentro de una investigación realizada en la Universidad UNIACC como parte de los fondos concursables año 2018, para docentes en función de la mejora continua de la praxis académica. Mencionada investigación titulada Estudio Exploratorio sobre Percepciones de Estudiantes de Periodismo y Publicidad jornada diurna de UNIACC en relación a las claves del éxito del desempeño docente para el logro de aprendizajes significativos en el aula, tuvo como eje central reconocer las habilidades blandas que los estudiantes de la UNIACC perciben y valoran como fundamentales al momento de evaluarnos de manera positiva en el aula. Utilizando una metodología mixta se logró identificar 19 habilidades blandas que fueron bipartidas en habilidades sociales y de comunicación que nuestro rol óptimo como docente requiere.

Palabras clave: UNIACC, educación universitaria, habilidades blandas, habilidades sociales y de comunicación

\begin{abstract}
This essay is part of an investigation carried out at UNIACC University in the framework of 2018 competitive funds for professors, based upon the continuous improvement of the academic praxis. The abovementioned research entitled "Exploratory Study on Perceptions of UNIACC Journalism and Advertising students in relation to successful keys in teaching performance for a significant learning achievement," had as a central axis the recognition of soft abilities perceived and valued by UNIACC daytime students when they assessed us in a positive way in the classroom. By using a mixed methodology, it was possible to identify nineteen soft skills, bipartited into social and communication skills, required for an optimal teaching role.
\end{abstract}

Keywords: UNIACC, college education, soft skills, social and communication skills 


\section{Introducción}

Lo que vemos cambia lo que sabemos,

lo que sabemos cambia lo que vemos

J. Piaget

En la actualidad, la labor y el desempeño de un docente en el aula debería perseguir siempre un aprendizaje significativo de los contenidos y, al mismo tiempo, resultados de aprendizaje que encierran su plan de asignatura por parte de su alumnado, es por esto que los diversos docentes académicos universitarios despliegan una serie de métodos y estrategias para lograrlo, tanto a nivel del uso de herramientas tecnológicas necesarias para enfrentar los diversos contenidos que dictan, como en metodologías que resultan o les han resultado exitosas, así como también de sus propias habilidades denominadas en la actualidad como habilidades blandas. En la actualidad, asistimos a un escenario tal que no es posible sostener la formación integral de nuestros alumnos sólo en el traspaso de contenidos e información. Vivimos plagados y saturados de información, la entrada de internet y la emergencia de la comunicación digital han hecho que el rol del docente en el aula de las carreras de formación presencial y modalidades online cobre cada día más valor.

Sin embargo, el éxito o no del rol docente y de su función muchas veces no se encuentra asegurado, en principio porque la mayoría de los docentes de nuestras aulas son profesionales que no disponen de herramientas de comunicación y un set de habilidades blandas, puesto que no fueron parte de su formación profesional, ni de la pedagógica implicada. A pesar de que existen diversos indicadores dentro de nuestra Universidad sobre la labor que cada docente efectúa en sus clases, mediante las encuestas de evaluación docente aplicada a los estudiantes, éstas resultan ser un dato cuantitativo que no se condice muchas veces con la realidad efectiva percibida por parte de los mismos alumnos.

Otro factor a considerar es el perfil de los estudiantes que encontramos en la actualidad en nuestras aulas, ellos son los denominados millenials, los cuales presentan ciertas características que desconocemos, o no conocemos del todo y que vienen a revolucionar los métodos de aprendizaje y la forma que tenemos, hasta el momento, los docentes de abordarlo.

Es por la razón recién mencionada que es posible visualizar un vacío de conocimiento al respecto, en tanto se desconocen: ¿cuáles son las habilidades que en la actualidad esperan los alumnos que los docentes desplieguen, cuáles les importan más?; ¿qué rol específico cumple un docente para sus alumnos en el aula?; ¿qué tipo de valores esperan los alumnos de sus docentes para ser considerados buenos docentes?: ¿qué tipo de características formativas de un docente ellos esperan encontrar y, al mismo 
tiempo, los influencian más?; ¿cuáles los motivan en su aprendizaje significativo?;¿qué es lo que hace que un docente tenga éxito en su rol y labor académica?

En consecuencia el estudio que se presenta a continuación, planteó como objetivo general el reconocer los elementos centrales que hacen que un docente sea percibido como un buen docente por parte de los alumnos de las carreras de Publicidad y Periodismo en la actualidad, al mismo tiempo que como objetivos específicos se tuvo: el identificar las habilidades que son percibidas por los alumnos en la actualidad como exitosas en sus docentes, identificar las habilidades blandas que un docente debería tener para ser percibido como un buen docente y lograr en consecuencia un aprendizaje significativo, y, por último generar uno o más talleres que permitan a los docentes de las carreras de Publicidad y Periodismo la emergencia de habilidades y herramientas de labor docente efectiva.

En cuanto a la metodología que fue abordada para el logro de los objetivos recién expuestos, se pensó en una metodología mixta que implicó realizar encuestas y focus para los alumnos respectivos. A este respecto, se debe considerar que para que un aprendizaje sea significativo, el sujeto debe percibirlo como tal y, al mismo tiempo, debe mostrar conductas acordes a ésta percepción, en consecuencia, mediante la metodología aplicada en campo a través de los instrumentos de encuesta y focus groups habrán diversas preguntas que indaguen en éste ítem particular, considerando la motivación que el docente es capaz de generar, la actitud hacia lo aprendido, la ampliación de conocimientos que él percibió debido a la labor docente, la praxis o aplicación de lo aprendido entre otras.

Resulta importante reconocer la percepción que tienen los alumnos sobre lo que hace que un docente consiga motivarlos y, en consecuencia lograr un aprendizaje significativo en ellos, siendo el aprendizaje significativo producto de una actitud o de lo que sienten y creen los alumnos más que del tipo de contenidos e información que se les entrega.

\section{Aproximaciones teóricas}

En relación al estudio realizado y lo que en éste se plantea se concentró el cuerpo teórico principalmente en la teoría constructivista del aprendizaje significativo descrita por Ausubel, Piaget y Vigotsky desde el punto de vista comunicacional. Para esto es necesario considerar que en la base de todo aprendizaje significativo se encuentra como motor o drive la variable motivación, es de esta manera, que se tiene como hipótesis central que el docente es capaz de tener un rol activo y principal en la generación y mantención de la motivación en sus estudiantes, a través del papel de líder de opinión que opera en el aula y en la relación que en ésta se realiza 
Como se ha establecido dentro de la Psicología; el aprendizaje depende de la atención, de la motivación y de la memoria para que se establezca asimilación y adecuación de la experiencia (Piaget,1983) pero para que esto ocurra y sea significado por alguien y se retroalimente construyendo nuevo conocimiento, lo que se llama aprendizaje significativo, debe existir un alguien que lo inicie, un emisor, o la fuente primera del circuito de la comunicación. Esta fuente primera dentro de nuestro contexto es el docente que en su rol de comunicador tiene que solventar ciertas características que han sido descritas como credibilidad, pero no son otra cosa que, un conjunto de habilidades blandas que hacen que soporte y comporte ese papel tan esencial. A saber la credibilidad de una fuente debe contar con cinco características: 1. Confiabilidad, 2. Conocimientos especializados, 3. Estatus o prestigio, 4. Simpatía o empatía, 5. Diversidad de rasgos físicos o semejanza con la audiencia (Loudon y Della Bitta, 1995).

Para el logro del aprendizaje significativo, que es lo que se pretende en la actualidad debemos entender a éste en su sentido más lato, a saber; según Ausebel (2002) un tipo de aprendizaje en que un estudiante relaciona la información nueva con la que ya posee; reajustando y reconstruyendo ambas informaciones en este proceso. Dicho de otro modo, y retomando incluso lo ya dicho por Piaget hacia los años cincuenta: la estructura de los conocimientos previos condiciona los nuevos conocimientos y experiencias, y estos, a su vez, modifican y reestructuran aquellos (Piaget, 1983). Este concepto y esta teoría se sitúan dentro de la denominada Psicología constructivista. El aprendizaje, según Ausubel (2002) se clasifica en distintas y articuladas categorías: intrapersonal, situacional, cognoscitivo y afectivosocial. La categoría intrapersonal se refiere a los factores internos. Incluye las variables de la estructura cognoscitiva, que son los conocimientos previos importantes para la asimilación de otra tarea de aprendizaje dentro del mismo campo. También considera la disposición del desarrollo, que se refiere a «la dotación cognoscitiva del alumno por la edad que tiene». Capacidad intelectual, se refiere a la aptitud y la capacidad de aprender derivada de su inteligencia general. Factores motivacionales y actitudinales son el deseo de saber, la necesidad de logro y de auto superación, y el interés. Factores de la personalidad, vinculadas al tipo de motivación, ansiedad y de ajuste personal. La categoría situacional incluye la práctica que se refiere a la frecuencia, distribución y condiciones generales. El ordenamiento de los materiales de enseñanza que incluyen la función de cantidad, dificultad, dimensión de los procesos, lógica interna, secuencia, velocidad y uso de apoyos didácticos. Ciertos factores sociales y de grupo como el clima psicológico del aula, cooperación y competencia, el estrato social, segregación racial y marginamiento cultural. Características del profesor en función de sus conocimientos, competencias 
cognoscitivas, conocimiento de la materia, competencia pedagógica, personal y actitudinal. La categoría cognoscitiva incluye los factores intelectuales objetivos, las variables de la estructura cognoscitiva, la disposición con respecto al desarrollo, la capacidad intelectual, la práctica y los materiales didácticos. La categoría afectivosocial remite a la motivación, actitudes, personalidad, factores de grupo y sociales y las características del profesor.

Finalmente, se puede sostener, desde lo teórico y conceptual que un aprendizaje significativo se evidencia en los siguientes aspectos: motivación por parte del estudiante por conseguir la aprehensión de lo impartido en el aula; motivación por ampliar la aprehensión de lo aprendido en el aula; inquietud y curiosidad por explorar nuevos conocimientos; actitud positiva hacia el conocimiento y el quehacer profesional específico, y ; aplicación de lo aprendido en el aula a su quehacer académico y profesional.

Ahora bien, el modelo teórico clásico de enseñanza-aprendizaje ha cambiado, se ha reformulado y en esta dirección surge un modelo que considera y asume al estudiante como un ser constructor del conocimiento. En este sentido, se plantea que una parte sustantiva del aprendizaje se da a través del quehacer, del practicar, de aplicar en la vida real lo que aprendemos en el salón de clases, por lo que la experiencia que la Universidad entrega, a través de la comunicación que se produce mediante un docente en el aula, resulta fundamental. Se entiende entonces por aprendizaje significativo; aquel que relaciona información nueva con la que ya se tiene obtenida de la experiencia y el contexto y para el cual se requiere de la motivación y en consecuencia de un actor (el docente) que imprima dicha motivación (Ausubel, 2009). Tal y como se describió con Piaget anteriormente, el nuevo conocimiento modifica la estructura cognitiva potenciando los esquemas que posibilitan la adquisión y construcción de nuevos y posteriores conocimientos.

Por otra parte, desde la ontología del lenguaje (Echeverría, 1995), se sostiene que el hombre es un ser pensante/hablante y cultural, de esta manera este ser está sistematizado, obedece y acata modos y modas, formas, es parte de un sistema cultural, está normalizado por leyes y modelos y socializa para poder vivir. Todo esto, forma la cultura del ser y de acuerdo a ella éste asume actitudes que son comportamientos o acciones frentre a la realidad. Es por esta razón por la que se hace indispensable conocer la cultura en la cual se da nuestra comunicación para que esta sea efectiva sobre todo si consideramos que la realidad se construye sobre la cotidianeidad y las intercaciones con el resto de nuestra comunidad (Berger y Luckman, 2003). 
Se puede concordar entonces que la comunicación y la educación son el mismo fenómeno, puesto que el proceso educativo es un proceso comunicativo donde el docente y el estudiante participan activamente en la adquisición de nuevos conocimientos (Freire, s.f.). Dentro de los elementos de la comunicación que debemos considerar existe un emisor, un mensaje, una situación, interferencias, retroalimentación, un canal y un receptor. De acuerdo a esto el mensaje es encodificado y decodificado, para ello necesita de ciertas códigos (Pozo, 2001).

Por otra parte, debemos atender siempre a la escucha activa de nuestros estudiantes en el aula, y ésta es una habilidad que se desarrolla con la experiencia y significa escuchar y entender la comunicación desde el punto de vista del otro. Para lo anterior se requiere disposición psicológica. Así como también para la empatía, el ponernos en el lugar del otro se hace fundamental para comunicarnos (Cano, 2005). En este escenario es en el que nos encontramos con las habilidades blandas que según Mujica Leiva (s.f.), se entienden como "el resultado de una combinación de habilidades sociales, de comunicación, de forma de ser, de acercamiento a los demás, entre otras, que hacen a una persona dada relacionarse y comunicarse de manera efectiva con otros". Es por tanto, un componente muy apreciado en la actualidad, pues posibilita el buen funcionamiento de las instituciones y de los equipos de trabajo. Así mismo el término habilidades sociales involucra las interrelaciones sociales entre las personas (Padilla, 1999), de esta forma las habilidades implican un conjunto de conductas aprendidas y lo social aporta a lo impersonal. Por su parte, Torres (1997) las define como un conjunto de comportamientos aprendidos que facilitan los refuerzos en situaciones sociales. Como seres sociales necesitamos de la satisfacción en nuestra interacción con los demás.

No son sólo un ingrediente en particular, sino que son el resultado de una combinación de habilidades sociales, de comunicación, de forma de ser, de acercamiento a los demás y otros factores que hacen que una persona pueda comunicarse de manera efectiva.

Por lo anterior es que las habilidades blandas tienen relación con lo que se conoce como inteligencia emocional; la relación y comunicación efectiva se ve afectada principalmente por la capacidad de conocer y manejar las emociones, tanto en nosotros mismos como en los demás, de acuerdo a cuánto conocimiento tengamos de los otros y de nosotros mismos (Goleman, 2008). También se puede utilizar el término de "competencias interpersonales" para agrupar a estas habilidades en una persona (Hofstadt, 2005).

En cualquier caso, tanto las habilidades blandas como duras pueden ser desarrolladas. En el caso de las habilidades duras el asunto es evidente - por ejemplo 
basta con leer el manual de funcionamiento de algo o ciertos textos para desarrollarlas en un cierto ámbito- mientras que las habilidades blandas necesitan de la voluntad y de la motivación para poder desarrollarlas y lograrlas (Mujica Leiva, s.f.).

Las habilidades sociales, a veces designadas como competencia social, no tienen una definición única, puesto que existe una confusión y diversidad conceptual (Hofstadt, 2005), entonces se puede decir que no hay consenso por parte de la comunidad científico-social; sin embargo, esto puede ser definido según sus principales características, y éstas señalan que las habilidades sociales son un conjunto de conductas aprendidas de forma natural (y que por tanto pueden ser enseñadas), que se manifiestan en situaciones interpersonales, socialmente aceptadas (ello implica tener en cuenta normas sociales y normas legales del contexto sociocultural en el que se actúa, así como criterios morales), y orientadas a la obtención de reforzamientos ambientales (refuerzos sociales) o autorrefuerzos. A su vez Hofstadt (2005) agrega que independientemente del contexto en el que se produzca la interacción, se considera socialmente habilidosa a aquella conducta incrementa y mejora la efectividad de los objetivos planteados y de las relaciones interpersonales, así como también la mejora del autoestima. Al mismo tiempo, Caballo (1993) define el concepto de habilidades sociales como el conjunto de conductas realizadas por un individuo en un contexto interpersonal en el que expresa sentimientos, actitudes, deseos, opiniones o derechos de un modo adecuado a la situación, respetando esas conductas en los demás, y donde generalmente resuelve los problemas inmediatos de la situación mientras reduce la probabilidad de que aparezcan futuros problemas.

Dentro de las habilidades sociales definidas por diversos autores se tiene: Saber escuchar; Iniciar una conversación; Mantener una conversación; Formular una pregunta; Dar las gracias; Presentarse y hacer presentarse a otras personas; Empatía; Dialogar; Pedir ayuda; Participar; Dar instrucciones y Seguir instrucciones; Disculparse; Convencer a los demás; Dar tu opinión sobre lo que piensas al respecto; Conocer los propios sentimientos; Expresar los sentimientos; Comprender los sentimientos de los demás; Enfrentarse con el enfado de otros; Expresar afecto; Resolver el miedo; Auto-recompensarse; Respetar los sentimientos; Consolar al afligido; Animar al decaído; Ayudar a los demás; Negociar; Emplear el autocontrol; No entrar en peleas (Rosales y otros, 2013).

Por otra parte y dentro de las habilidades sociales que han sido dispuestas en la literatura respectiva y que, en la actualidad podemos decir que concuerdan en mucho con los resultados de esta investigación que se presenta, podemos encontrar 4 tipos, a saber: Cognitivas, todas aquellas habilidades en las que intervienen aspectos psicológicos; las Emocionales, son aquellas habilidades en las que están implicadas la 
expresión y manifestación de diversas emociones, es decir lo que se siente. La ira, el enfado, la alegría, la tristeza; las Instrumentales, se refiere a aquellas habilidades que tienen una utilidad, o sea lo que se hace. Por ejemplo, negociación de conflictos y las Comunicativas, que se refieren a aquellas habilidades en las que interviene la comunicación, o sea lo que se dice. Por ejemplo, formular preguntas, iniciar y mantener conversaciones, entre otras.

Para finalizar con nuestra aproximación teórica, no podemos olvidar el contexto en el que ocurre el proceso enseñanza-aprendizaje que es nuestra cultura. En la denominada era de la información y de los avances tecnológicos así como también de la denominada globalización, asistimos a una revolución del conocimiento (Castells, pero, esta revolución no solo atañe al conocimiento en sí mismo sino también a la construcción de subjetividades (Elliot, 1995) diversas que viven y se construyen en la inmediatez del tiempo y en la virtualidad del espacio vivido (Sartori, 1997). La era del vacío denominada así por Lipovetsky en1986 es también la era de la liquidez como denuncia Bauman algunos años más tarde, en la cual todos los valores e ideales sólidos que sostenían la subjetividad moderna se vuelven cada vez más fluidos e inmediatos en un escenario de constante espectáculo (Debord, 1999) y simulacros (Baudrillard, 1998). En estos tiempos es que surgen los millenials como categoría subjetiva, los cuales presentan ciertas características que la Psicología aun no logra dimensionar en sus aspectos generales y específicos. Son los jóvenes actuales, los que padecen de falta de compromiso, de sentido crítico, los que padecen de angustia, los que evidencian un pensamiento denominado blanco según Green (1987), los que presentan debilidad en el sostén de sus creencias, son lights frente a todo: a su educación, a su formación, a sus carreras profesionales, a su consumo, a su vida en general carente de futuro (Sinek, 2016). Viven exigiendo derechos pero se muestran sin deberes, falta de sentido político y ciudadano es más que evidente, para ellos la participación social se ha vuelto un espacio privado e individual.

Es de esta manera como Castells (1998) nos recuerda que debido al proceso globalizador se ha producido una "oleada" de expresiones de identidad colectiva que desafían la globalización en defensa de la singularidad cultural y el control sobre la propia vida y el medio ambiente.

De esta necesidad de comenzar a comunicarnos en red, se han abierto más canales de comunicación, por ende, más tráfico de mensajes; así surge la Aldea Global, que sugiere que "el advenimiento de las tecnologías de información ha venido marcado por una necesidad social de comunicación, pero que el desarrollo acelerado de estas mismas tecnologías ha logrado transformar y modelar las formas de comportamiento humano. En fin, una relación recursiva". (Arcila, 2010, p. 56). Autores como Moreno 
(2000) sostienen que la sociedad del conocimiento representa la fase más avanzada de los cambios impulsados por la globalización en las últimas décadas, y conjuntamente con la internacionalización.

Desde este espacio es que también ha surgido el postmillenial, que es aquel nacido después de 1995 y que presenta ciertas características que hacen interesante que las identifiquemos con el fin de poder conocer un poco mejor a nuestros estudiantes. Son los verdaderos nativos digitales, no conciben un mundo si wi-fi, youtube o instagram, pueden usar hasta cinco dispositivos a la vez y son muy dependientes de la tecnología. Por otra parte son más recelosos con su intimidad, saben que no deben compartirlo todo y, al mismo tiempo, son más conscientes de los riesgos e inconvenientes que ello implica. Se presentan como autosuficientes, autodidactas e informados. Están habituados a realizar múltiples tareas. Y por último, como característica a rescatar tienen su propio vocabulario, demuestran mayor activismo social y se prestan a mayor número de voluntariados (lavanguardia.com).

Todas estas características hacen que debamos repensar las Instituciones encargadas de socializar, entre ellas la Universidad, y en consecuencia traspasar la solidez perdida y aprender de estos nuevos tiempos en lo que Concheiro (2015) llama la filosofía de la inmediatez. Como ya se ha mencionado, dentro de las Instituciones que debemos reformular como sociedad se encuentra la Universidad, que recibe estas subjetividades sin, hasta el momento, clara definición ni metodologías claras para posibilitar relaciones efectivas. Es difícil saber qué estamos haciendo bien, o más aún, cómo estamos enfrentándonos a nuestros estudiantes, qué quieren ellos a través de nuestro rol como docentes, cómo poder formarlos de una manera adecuada a lo que ellos desean recibir para así, sentirse parte de nuestra sociedad. Cómo logar una educación inclusiva e integral en su más pleno sentido si no sabemos a quién le hablamos y con quién nos comunicamos y las expectativas que tienen de nuestro rol.

\section{Marco metodológico del estudio realizado}

Para abordar este estudio se utilizó una metodología mixta, en la cual se realizaron cuatro focus groups a los estudiantes de Publicidad y Periodismo, ambos en modalidad presencial y diurna para recabar y levantar la información necesaria en pos de construir una encuesta con las habilidades relevantes para ellos. En principio se pensó en la construcción de muestras probabilísticas con el registro de los alumnos y en una muestra no probabilística para los focus groups, sin embargo dado que no era posible obligar a los estudiantes a participar, se realizaron ambos instrumentos con la población indicada y con muestras no probabilísticas. Obteniendo el 90\% aproximado de participación. En cuanto al número de focus groups pensados se cree 
que con un focus aplicado a estudiantes en primer y segundo año de cada carrera y otro focus aplicado a estudiantes que cursen tercer y cuarto de las carreras en cuestión sería suficiente, considerando que no se muestran mayores cambios en estos años. O sea se aplicó un total de 4 focus groups. A continuación se presentan los diseños de instrumentos y los resultados consecuentes de los focus groups para la elaboración, a su vez, de la encuesta. En cuanto a los objetivos de los focus groups se tuvo: describir la percepción sobre lo que es un buen docente para los estudiantes de UNIACC; Describir las habilidades de lo que los estudiantes denominan un buen docente; Describir la percepción de los alumnos sobre el significado de aprendizaje significativo; Identificar las habilidades que son percibidas como favorables para un aprendizaje significativo en el aula e Identificar las habilidades que son percibidas como desfavorables para un aprendizaje significativo

\section{Principales resultados obtenidos}

La muestra de los focus groups estuvo constituida por 40 alumnos de las carreras de Publicidad y Periodismo modalidad presencial y jornada diurna. Obteniéndose 21 alumnos de Publicidad y 19 de Periodismo. Se les convocó de manera voluntaria por medio de los correos institucionales. La respuesta fue alta a esta convocatoria, ya que participaron alrededor del 50\% de ambas carreras.

En primer lugar, se tiene que para los estudiantes la educación en el aula va mucho más allá de transmitir y ser capaz de transmitir contenidos sino que tiene que ver con un valor de formación, un valor integral donde el rol del docente es fundamental. Ahora bien, la percepción que tienen los alumnos en general de lo que es un buen docente tiene directa relación con la cercanía que muestre y, a su vez, la relación que genere con sus estudiantes.

Otro factor relevante a considerar, en la percepción de los estudiantes de ambas carreras es la motivación que muestre un docente tanto en su rol como docente, esto es en su praxis, así como en el aprendizaje de sus estudiantes. De la misma forma, los profesores que son percibidos como malos profesores son aquellos que no motivan a sus estudiantes.

Finalmente, los alumnos rescatan el factor compromiso. Este compromiso, el que un docente muestra hacia los estudiantes y, en particular hacia el grupo curso, es vivenciado por ellos en diversas actividades como por ejemplo en el simple hecho de venir a hacer clases. En este sentido, los alumnos recalcan que el buen docente es aquel que es capaz de identificar sus características e intereses y conduce el aprendizaje hacia el logro de una meta común. 
En cuanto a las habilidades percibidas por los estudiantes como deseables, es posible establecer que, en general un buen docente debe ser aquel que muestre, además de sus habilidades duras y su conocimiento sobre la materia que enseña, habilidades que son parte de la comunicación y de las habilidades sociales. Al mismo tiempo, la comunicación que sea capaz de establecer se torna del todo relevante y es parte de la relación que se establece en el aula y en este sentido dicen que Interactuar - eso es fundamental. En este sentido un docente debe ser confiable, empático, cercano, al mismo tiempo que debe evidenciar conocimiento y ser veraz. En su lenguaje debe tener buena dicción, y un correcto uso del lenguaje con un buen vocabulario y finalmente ser creíble.

Finalmente y en relación a las habilidades específicas, se rescataron 19 habilidades que fueron dispuestas en la encuesta que se les realizó a los alumnos. Teniendo un alto porcentaje de participación del universo consultado.

Por otra parte, los resultados que se obtuvieron con la aplicación de la encuesta que estuvo conformada por estudiantes de segundo, tercer y cuarto año de las carreras de Publicidad y Periodismo modalidad diurna y presencial se muestran como parte de las conclusiones más abajo detalladas. Sin embargo, cabe agregar que la muestra de la encuesta aplicada fue dada según quienes respondieron del Universo total de mencionados estudiantes, en este sentido se constituyó como una muestra no probabilística por conveniencia del investigador y a juicio de experto. El total del universo era de 44 estudiantes de Publicidad y 44 estudiantes de Periodismo diurno. Del total de 88 estudiantes, 70 responden la encuesta, es decir, un 80 por ciento. Encontrándose un 60 por ciento de estudiantes de Publicidad y un 40 por ciento de Periodismo diurno.

\section{Conclusiones}

De las 19 habilidades levantadas en los focus groups se obtiene el siguiente análisis. En primer lugar, se separarán las habilidades en dos grandes grupos, de acuerdo a la literatura contemplada y revisada, al mismo tiempo que considerando lo expresado por los alumnos y analizado dentro del contexto de los focus groups realizados, así como también de acuerdo al criterio y conocimientos del investigador. De acuerdo a ello se conceptualizó cada una de las habilidades, obteniéndose lo siguiente:

1. Habilidades comunicacionales: es aquel grupo de habilidades que se centran en la eficacia del emisor y el logro en la transmisión del mensaje, lo que provoca una recepción exitosa de un determinado mensaje y en consecuencia una comunicación efectiva. Para nuestros estudiantes lo que hace que un docente sea un buen docente tiene que ver con que sea un buen comunicador, en este sentido se rescatan las 
habilidades de credibilidad, la asertividad, el liderazgo, el dinamismo y el uso de un buen lenguaje tanto verbal como paraverbal.

1.1. Credibilidad: se define como aquella habilidad que sostiene una persona al momento de emitir un mensaje determinado. En este sentido, alguien es creíble cuando logra transmitir de manera efectiva y de manera veraz un determinado mensaje. La credibilidad, para nuestros estudiantes también se sustenta en cierta semejanza con ellos, en el expertisse que se demuestre, en la empatía y en el carisma. Esta habilidad obtuvo en la encuesta un $76 \%$ de las respuestas muy importante y un $24 \%$ que la considera importante.

1.2. Asertividad: se define como aquella habilidad de decir lo correcto en el momento indicado y de manera correcta. Es decir que el mensaje sea claro en sus códigos verbales y no verbales y que considere al mismo tiempo el contexto del emisor y en el cual es emitido. En relación a esta habilidad el 51\% de los encuestados la considera muy importante mientras que un $49 \%$ que la considera importante.

1.3. Liderazgo: definida como aquella capacidad de conducir a un grupo de manera exitosa hacia el logro de uno o más objetivos. El liderazgo se sustenta en el poder que ejerce el líder en relación a los demás miembros del grupo y la aceptación de ese poder por parte del grupo. Para los alumnos forma parte de la autoridad y el rol que tiene un docente dentro del aula pero no se debe confundir con autoritarismo. El 50\% de los encuestados considera muy importante el liderazgo en un docente versus un $40 \%$ que la considera importante y un $10 \%$ poco importante.

1.4. Confianza-seguridad: se define como la habilidad que tiene un docente para transmitir seguridad y confianza en lo que dice y expresa. Tiene que ver con la credibilidad también y en mucho con las habilidades de comunicación persuasiva y efectiva que alguien es capaz de mostrar hacia el resto. El 69\% de los encuestados considera muy importante la confianza/seguridad en un docente versus un $27 \%$ que la considera importante y un $4 \%$ poco importante.

1.5. Buen lenguaje verbal y paralingüístico: esta habilidad queda definida como el buen manejo y uso del lenguaje en todo sentido y en todos sus tipos. Lenguaje verbal y paraverbal. Hablamos de un lenguaje que es formal, instruído pero a la vez cercano. Nuestros estudiantes valoran el hecho que un docente muestre un nivel superior de lenguaje al utilizarlo con ellos en el aula y lo que se desea transmitir. El 67\% de los encuestados considera muy importante esta habilidad en un docente mientras que un $30 \%$ la considera importante y sólo un $3 \%$ poco importante. 
1.6. Dinamismo: queda definida como aquella habilidad que hace que la clase de un docente sea interesante, motivadora y atractiva. Un docente es dinámico cuando ofrece diferentes metodologías y formas de enseñar lo que debe enseñar, pero también es dinámico cuando tiene una proxémica adecuada y un uso del espacio adecuado además de un tono de voz poco monótono y activo. En relación a esta habilidad el $63 \%$ de los encuestados considera muy importante el dinamismo en un docente versus un $34 \%$ que la considera importante y sólo un $3 \%$ poco importante.

2. Habilidades sociales: las habilidades sociales están centradas principalmente en las características personales y, las habilidades en este sentido, se remiten a cuestiones de orden individual y valórico. Para nuestros estudiantes son fundamentales en este sentido, el respeto, la adaptabilidad, la autocrítica, la integridad, la humildad, la empatía, la motivación, la honestidad, la tolerancia, la paciencia, el compromiso, la cercanía y la objetividad.

2.1. Respeto: se define como la base de la relación docente-estudiante. Y queda traducido en una actitud deferente hacia el estudiante en todo momento, lugar y situación. Para nuestros estudiantes en un $83 \%$ de los encuestados se considera una habilidad docente muy importante, mientras que un $14 \%$ la considera importante y sólo un 3\% poco importante.

2.2. Adaptabilidad: se define como aquella habilidad que tiene un docente al momento de escoger estrategias de aprendizaje ceñidas al conocimiento del grupo que tiene en frente. De acuerdo a lo mencionado es muy importante para los estudiantes que un docente sea capaz de conocerlos, de saber quienes son y adaptarse al contexto grupal y sociocultural en el que se encuentra ubicado. En este sentido, el $49 \%$ de los encuestados considera muy importante la adaptabilidad en un docente versus un $49 \%$ que la considera importante y un $3 \%$ poco importante.

2.3. Autocrítica: se define como aquella habilidad que implica la capacidad de admitir cometer un error o ser ignorante en un determinado momento sobre un tema en específico y, al mismo tiempo, darse cuenta de ello y resolver la situación. Tiene relación con admitir los errores que uno pueda cometer tanto en materia de conocimientos como en el plano evaluativo-académico y también personal. En este sentido, se obtuvo que un $67 \%$ de los encuestados considere muy importante la autocrítica en un docente mientras que un $29 \%$ la considere importante y un $4 \%$ poco importante.

2.4. Integridad: se refiere a aquella habilidad blanda que muestra un pleno uso, control y sapiencia de los valores de la sociedad que quedan demostrados en una personalidad coherente y consistente. Esto para los estudiantes queda de manifiesto 
muchas veces a través del ejercicio en el aula y en su ámbito profesional en específico. Un $60 \%$ de los encuestados considera muy importante la integridad en un docente versus un $37 \%$ que la considera importante y sólo un $3 \%$ poco importante.

2.5. Humildad: es aquella habilidad personal que muestra un docente frente a lo que sabe y a lo que no sabe también. Es mostrarse humilde y no narcisista frente a los estudiantes, sobre todo en lo que respecta a los logros y títulos obtenidos en el trayecto de la vida. En este sentido un $44 \%$ de los encuestados considera muy importante la humildad en un docente versus un $46 \%$ que la considera importante y un $10 \%$ poco importante.

2.6. Empatía: se define como aquella habilidad que tiene un docente de ponerse en el lugar del otro y saber escuchar al otro comprendiendo la situación de éste mismo. Para un $60 \%$ de los encuestados la empatía se considera muy importante mientras que un $30 \%$ la considera importante y un $10 \%$ poco importante.

2.7. Motivación: se define como aquella habilidad que tiene un docente para primero, mostrar pasión frente a su ejercicio docente y segundo, poder traspasar esa energía a sus alumnos en pos de fomentar el aprendizaje tanto en el aula como fuera del aula. En este sentido, un $74 \%$ de los encuestados considera muy importante la motivación en un docente versus un $19 \%$ la considera importante y un $7 \%$ poco importante.

2.8. Honestidad: se define como aquella habilidad valórica que los alumnos perciben en un docente al tratar la veracidad como principio de la relación docente-estudiante. En este sentido, un $69 \%$ de los encuestados considera muy importante la honestidad en un docente, así como un 30\% la considera importante y un solo un $1 \%$ como poco importante.

2.9. Tolerancia: se define como aquella habilidad de un docente para aceptar y respetar las opiniones de sus estudiantes sin juicios de valor. En este sentido, los estudiantes requieren de un docente que no prejuzgue sus conocimientos así como sus valores, creencias e ideas. La encuesta realizada revela que un $64 \%$ de los encuestados considera muy importante la tolerancia en un docente versus un $36 \%$ la considera importante.

2.10. Paciencia: se define como aquella habilidad de un docente para tener y expresar calma en relación a los aprendizajes que van mostrando sus estudiantes. Ser capaces de experenciar con ellos el recorrido, el proceso y los avances, para nuestros estudiantes es fundamental. A este respecto un $61 \%$ de los encuestados considera muy importante la paciencia en un docente mientras que un $31 \%$ la considera importante y un $7 \%$ poco importante. 
2.11. Compromiso: se define como aquella habilidad que nos habla de un docente que es responsable con su ejercicio docente así como de las relaciones personales y académicas que genera y sostiene con sus estudiantes. También es la capacidad de tomar conciencia de la importancia que tiene el docente en su rol de docente en virtud de los resultados de sus estudiantes. El compromiso es fundamental en tanto considera tanto el hecho de venir a hacer clases como hacerlas bien. Para los estudiantes el compromiso es una virtud y un valor en un buen docente. En este sentido, los datos de la encuesta son reveladores, un $80 \%$ de los encuestados considera muy importante el compromiso en un docente mientras que un $20 \%$ la considera importante.

2.12. Cercanía: es aquella habilidad que tiene un docente para generar un lazo afectivo con sus estudiantes. Este lazo afectivo no debe ser obligado ni tampoco informal, sino que es aquella habilidad que muestra un docente para ser percibido como alguien en quien se puede confiar dentro y fuera del aula, así como también en términos académicos y personales. Un $41 \%$ de los encuestados considera muy importante la cercanía en un docente vs un $46 \%$ la considera importante y un $13 \%$ poco importante.

2.13. Objetividad: se define como aquella habilidad de neutralidad en un docente. Es decir el docente tiene la habilidad de mostrarse con un agente neutral frente a todos sus alumnos, no diferenciando en aspectos de género, físicos, mentales, ni etarios ni socioculturales. Para nuestros estudiantes ser objetivo implica tener neutralidad en las evaluaciones y en el trato personal que se les da a todos los estudiantes. No mostrando en ninguna ocasión preferencia o discriminación por alguien o por algo. Un $71 \%$ de los encuestados considera muy importante la objetividad/neutralidad en un docente mientras que un $28 \%$ la considera importante y sólo un $1 \%$ poco importante.

Finalmente, se puede concluir que en relación al objetivo general de este estudio que era reconocer los elementos centrales que hacen que un docente sea percibido como un buen docente por parte de los alumnos de las carreras de Publicidad y Periodismo en la actualidad, se tiene que son un conjunto de habilidades tanto sociales como comunicacionales, algunas duras pero principalmente blandas las que hacen que un docente sea reconocido de esa forma por ellos. Las más destacadas e identificadas por este estudio exploratorio fueron principalmente las ya descritas, sin embargo es posible pensar que en todas ellas hay un conductor general que hace que un docente sea percibido como un buen docente y es su calidad humana, al mismo tiempo que esa calidad humana expresada en su profesionalismo, responsabilidad y compromiso hacia sus estudiantes y el contexto general de la universidad, lo que produce en ellos 
una carga afectiva importante y el factor motivacional que es motor de todo aprendizaje significativo. El docente es aquel que los modela y los motiva y que, a la vez, les permite avanzar en su perfeccionamiento y desarrollo integral.

\section{Referencias}

Abélès, M. (2008). Anthropologie de la globalisation. Francia: Payot.

Arcila, C. (2010). La presentación del sí-mismo en los entornos virtuales Comunicación, alteridad e identidad en chats, blogs y redes sociales (Tesis doctoral). Universidad Complutense de Madrid, Madrid.

Ausebel, D. (2002). Adquisición y retención del conocimiento. Una perspectiva cognitiva. Madrid: Paidós Ibérica.

Ausubel, D., Novak, J., \& Hanesian, H. (2009). Psicología educativa: un punto de vista cognoscitivo. México: Trillas.

Bain, K. (2006). Lo que hacen los mejores profesores universitarios. Valencia: Publicacions Universitat de Valencia.

Ballester, R. et al (2002). Habilidades sociales. Evaluación y tratamiento. Madrid. Síntesis.

Baudrillard, J. (2000). Las estrategias fatales. Madrid: Anagrama.

Bauman, Z. (2011). Daños colaterales. Madrid: Fondo de Cultura Económica.

Bauman, Z. (2016). La modernidad líquida. Madrid: Fondo de Cultura Económica.

Berger, P. \& Luckmann, T. (2003). La construcción social de la realidad. Buenos Aires.: Amorrortu.

Bravo \& Cáceres. (2016). Didáctica y optimización del proceso de enseñanza aprendizaje. IPLAC. Impresión ligera. C. de La Habana.

Caballo, V. (1993). Manual de técnicas de terapia y modificación de conducta. Madrid: Siglo XXI.

Cano, E. (2005). Cómo mejorar las competencias de los docentes. Pontevedra: Grao.

Castells, M. (2001). La galaxia internet: reflexiones sobre internet, empresa y sociedad. Barcelona: Plaza y Janés. 
Castells, M. (2012). Comunicación y Poder. Madrid: Siglo XXI.

Castro, I. \& Moreno, L. (2006). El modelo comunicativo. Teóricos y Teorías relevantes. Trillas: México.

Di Lucca, S. (2013). El comportamiento actual de la Generación Z en tanto futura generación que ingresará al mundo académico. Recuperado de https://fido.palermo.edu/servicios_dyc/catalogo_investigacion/detalle_proyec to.php?id_proyecto=2255\&titulo_proyectos=El\%20comportamiento\%20actual\% 20de\%20la\%20Generaci\%F3n\%20Z\%20en\%20tanto\%20futura\%2ogeneraci\%F3n\%20 que\%2oingresar\%E1\%20al\%20mundo\%20acad\%Egmico

Díaz, F. (2010). Estrategias Docentes para un Aprendizaje Significativo. México: McGraw Hill Education.

Echeverría, R. (2005). La Ontología del Lenguaje. Santiago: LOM.

Galeano, E. (1998). Modelos de comunicación. Buenos Aires: Macchi.

Gismero, E. (1999). Escala de Habilidades sociales: Manual. Madrid: TEA Ediciones

Goleman, D. (2008). Inteligencia emocional. Barcelona: Kairós

Hernández, R., Fernández, C. \& Baptista, M. (2010) Metodología de la Investigación. México D.F.: McGraw-Hill Interamericana.

Hofstadt, C. (2005). El libro de las habilidades de Comunicación. España: Díaz de Santos

Knight, P. (2005). El profesorado de educación superior: Formación para la excelencia. Madrid: Narcea

Lipovetsky, G. (1986). La era del vacío. Madrid: Anagrama

Loudon \& Della Bitta. (1995). Comportamiento del consumidor. México: McGraw Hill

McLuHan, M. (1997). La aldea global. Barcelona: Gedisa.

McQuail, D. \& Windahl, S. (1997). Modelos para el estudio de la comunicación colectiva. Ediciones Universidad de Navarra SA.

Medina, J. L., Cruz, L., \& Jarauta, B. (2016) La dimensión dialógico-reflexiva del conocimiento didáctico del contenido de la docencia universitaria. Revista de Educación, 374. Octubre-Diciembre 2016, pp. 69-93 
Moreno, L. (2000). Sociedad del conocimiento y sustentabilidad de la globalización. Caracas: Nueva Sociedad.

Moya, M. (2016). Habilidades comunicacionales y comunicación política. (Tesis doctoral). Universidad Miguel Hernández. España. Recuperado de: http://dspace.umh.es/bitstream/11000/3208/1/TD\%20Moya\%20Ruiz\%2C\%20Maria \%20Teresa.pdf

Mujica, J. (2015). ¿Qué son las habilidades blandas y cómo se aprenden?: Habilidades Blandas $N^{\circ} 1$ Enero 2015. Recuperado de https://educrea.cl/que-son-lashabilidades-blandas-y-como-se-aprenden/

Piaget, J. (1976). La construcción de lo real en el niño. Buenos Aires: Nueva visión.

Piaget, J. (1983). El lenguaje y el pensamiento en el niño. Buenos Aires: Rústica.

Pozo, J. \& Pérez, M. (Coords.). (2009). Psicología del aprendizaje universitario: la formación basada en competencias. Madrid: Morata.

Pozo, J. (s.f.). Técnicas de Comunicación personal y grupal. Alicante: Club Universitario.

Prensky, M. (2001). Digital natives, digital immigrants part 1. On the horizon, 9(5), 1-6. Recuperado de http://www.marcprensky.com/writing/Prensky\%20\%20Digital\%20Natives,\%20Digital\%20lmmigrants\%20-\%20Part1.pdf

Regader, B., (s.f.). La teoría del aprendizaje de Jean Piaget. Psicología y Mente. Recuperado de https://psicologiaymente.com/desarrollo/teoria-delaprendizaje-piaget

Roca, G. (5 de agosto de 2007). Marc Prensky y los nativos digitales. [Artículo en un blog]. Recuperado de: http://www.genisroca.com/2007/08/05/marc-prensky-ylos-nativos-digitales/

Rosales, J., Caparrós, B. \& Molina, I. (2013). Habilidades sociales. Madrid: McGraw Hill Education.

Sánchez, U. (2006). Modelos y esquemas de comunicación. Medellín: Sello Editorial.

Sanz, G. (2005). Comunicación efectiva en el aula. Pontevedra: Grao.

Sartori, G. (1997). Homo videns: La sociedad teledirigida. Madrid: Taurus.

Torres, T. (1997). Habilidades sociales: Manejo de los problemas de conducta social en el hogar y las escuelas. Lima: Colegio B. F. Skinner. 
Watzlawick, P. (2014). No es posible no comunicar. Barcelona: Herder.

\section{Andrea Naranjo Lama.}

Psicóloga Universidad Diego Portales. Doctora en Psicología, Universidad Complutense de Madrid, España. Postítulo en Clínica diferencial de las neurosis, Universidad de Chile. Postítulo en Epistemología de las Ciencias, Universidad de Chile. Certificado de desarrollo cursos en modalidad online, Universidad Mayor. Diplomado en docencia universitaria, Universidad UNIACC. Docente de diversas asignaturas y carreras en Universidad UNIACC, académica de la Universidad de Santiago de Chile, departamento de Publicidad e Imagen y docente en Magister en Comunicación digital en Universidad Mayor. Candidata a Magister en Educación Superior en Universidad UNIACC.

Email: dra.naranjo@gmail.com 